\title{
Avoiding the bowel: a report of a mature cystic teratoma displaying fully developed intestinal tissue protruding from an ovarian tumor
}

\author{
David B. Nelson • Barbara L. Hoffman • \\ Yuri Lemeshev $\cdot$ Larry E. Word
}

Received: 30 October 2009 / Accepted: 23 November 2009 / Published online: 23 December 2009

(C) Springer-Verlag 2009

\begin{abstract}
We present a previously unreported case of a grossly visible, fully developed intestinal segment protruding from an ovarian teratoma.
\end{abstract}

Keywords Mature cystic teratoma $\cdot$ Intestine

\section{Introduction}

Ovarian teratomas arise from a single germ cell and may contain any three germ layers-ectoderm, mesoderm, or endoderm. Consequently, these tumors contain a haphazard collection of tissue such as hair, fat, bone, and teeth [1]. Recently, authors have identified interesting intestinal associations with ovarian teratomas. Kwon et al. reported a grossly visible, well-organized intestinal loop with mesentery arising in a mature cystic teratoma concurrent with a malignant mixed germ cell tumor and yolk sac tumor in the same ovary [2], and Tang et al. reported a mature cystic teratoma of the ovary containing complete colonic wall in continuity with an endocervical-type mucinous

Informed consent: obtained and signed by the patient

D. B. Nelson $(\bowtie) \cdot$ B. L. Hoffman · Y. Lemeshev $\cdot$ L. E. Word Department of Obstetrics and Gynecology,

University of Texas Southwestern Medical Center/Parkland

Health and Hospital System,

5323 Harry Hines Boulevard,

Dallas, TX 75235-9032, USA

e-mail: dbnels@parknet.pmh.org

D. B. Nelson • B. L. Hoffman • Y. Lemeshev • L. E. Word Department of Pathology, University of Texas Southwestern Medical Center/Parkland Health and Hospital System, 5323 Harry Hines Boulevard,

Dallas, TX 75235-9032, USA cystadenoma [3]. We now present a previously unreported case of a grossly visible, fully developed intestinal segment protruding from a mature cystic ovarian teratoma concurrent with a mucinous cystadenoma.

\section{Case report}

A 45-year-old, gravida 4, para 2, abortus 2, morbidly obese Hispanic female presented desiring permanent sterilization. Her past medical history was significant for hypertension and prior ectopic pregnancy treated via laparotomy. The patient was deemed an appropriate candidate for laparoscopic tubal sterilization. At the time of laparoscopy, significant pelvic adhesive disease was encountered, and a complex right ovarian mass of indeterminate size was discovered. Laparotomy was performed secondary to extensive adhesions. Findings were: (1) an approximate $12-\mathrm{cm}$ mass grossly consistent with a benign mature cystic teratoma, (2) a $10 \times 0.5 \mathrm{~cm}$ tubular structure, which exited the mass, blindly terminated into the greater omentum, and had the gross appearance of intestinal tissue and without communication to bowel, and (3) normal appearing appendix. The mass and appendage were completely excised at their respective margins. Filshie clips were applied to the right and left fallopian tubes. The patient's postoperative course was unremarkable.

Final pathologic analysis of the mass revealed a mature cystic teratoma, measuring $12 \times 13 \times 4 \mathrm{~cm}$, filled with yellow, greasy material, hair, and concurrent portions of a mucinous cystadenoma. A tubular structure, measuring $9 \mathrm{~cm}$ in length and $0.5 \mathrm{~cm}$ in diameter, was found protruding from the specimen with a grossly visible lumen on cross section (Fig. 1). The structure extended into the 


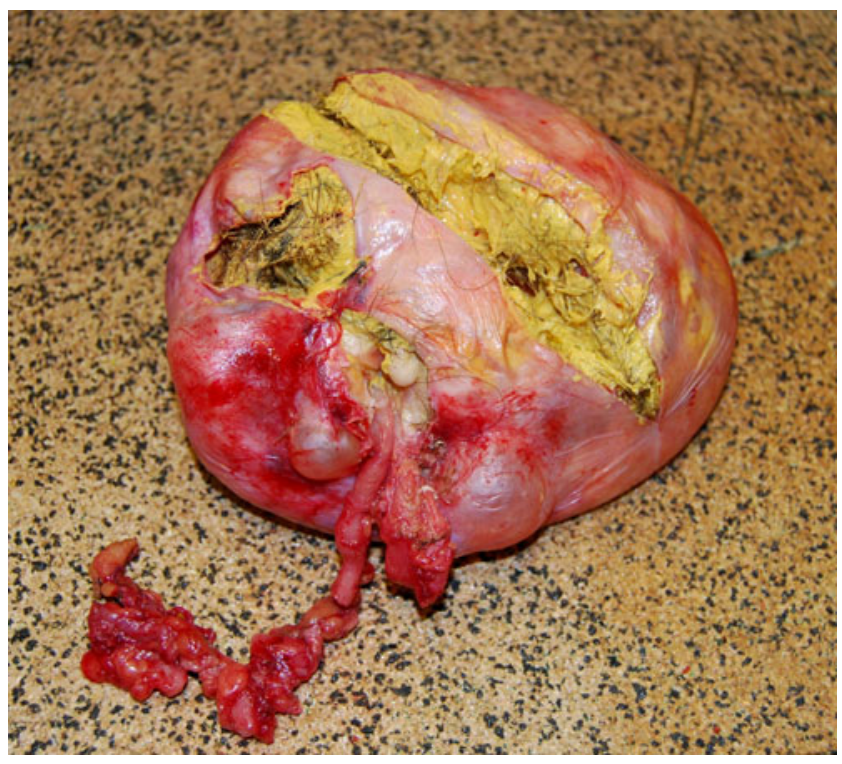

Fig. 1 a Macroscopic view of excised specimen including ovarian mature cystic teratoma with dissected intestinal tissue protruding from a dimple in the mass. Two slits on the top of the mass were created for frozen sectioning. Within these slits, bright yellow fatty cyst contents are mixed with hair

specimen cavity, where it blindly terminated with expansion to $1.9 \mathrm{~cm}$ in diameter (Fig. 2). Microscopically, the tubular structure, both inside and outside of the mass, represented well-formed colonic wall (Fig. 3) with myenteric (Auerbach's) nerve plexus located in the muscularis

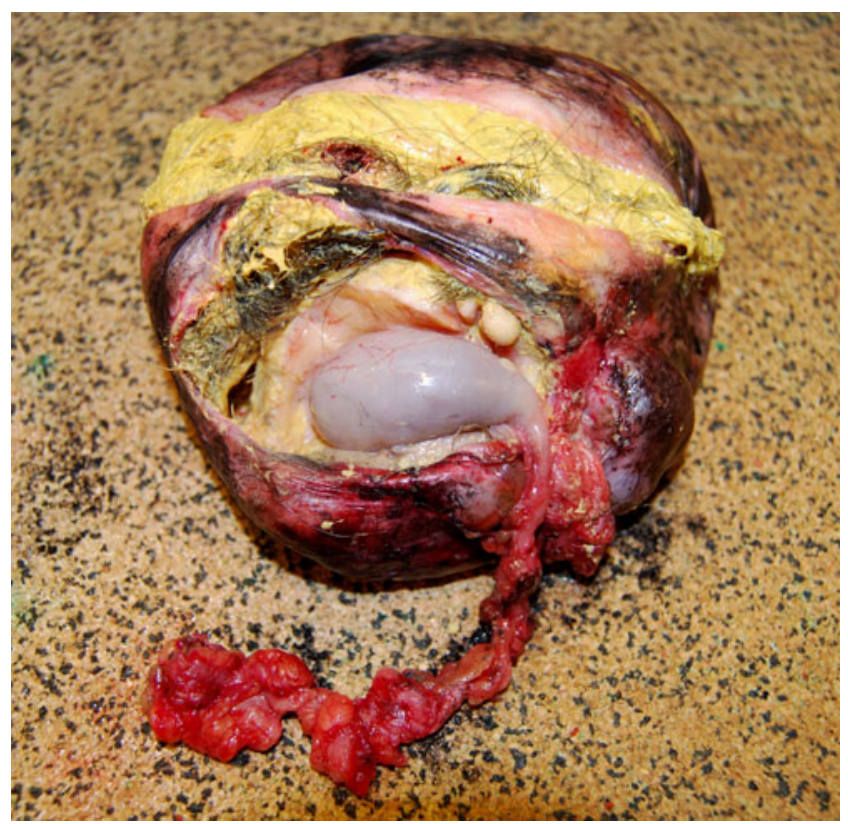

Fig. 2 The smaller external tubular structure extends into the teratoma. Within the cyst, it takes the form of a blind-ended dilated bowel loop

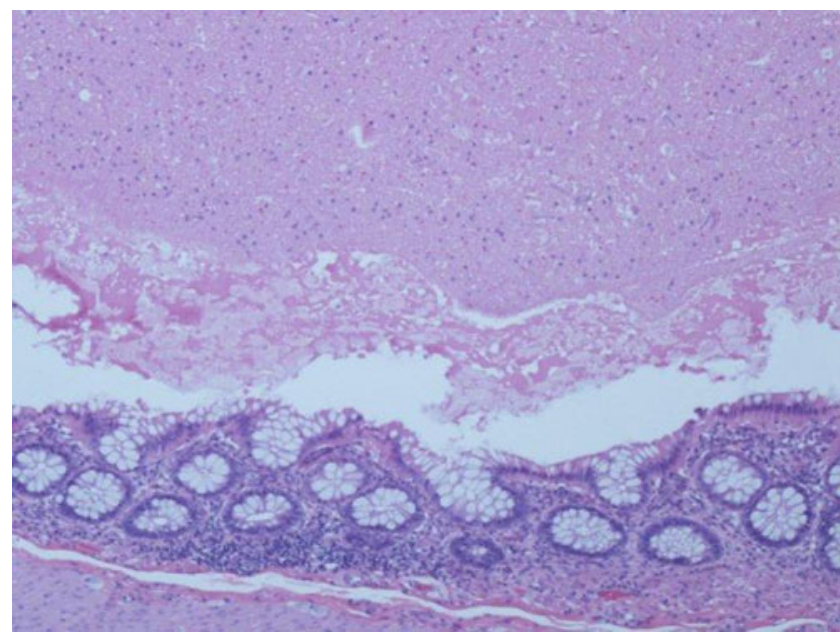

Fig. 3 Microscopically, the tubular structure representing well-formed colonic wall

propria (Fig. 4). The dermoid protuberance occupied 30 $35 \%$ of the cystic cavity and consisted of neural tissue, aggregates of ganglion cells, smooth muscle, cartilage, nerve trunks, bone marrow, bone, alveolar parenchyma, and skin with skin appendages. The wall of the mass was focally ulcerated and replaced with granulation tissue containing numerous foreign body giant cells.

\section{Comment}

Mature cystic teratomas comprise approximately $10 \%$ to $25 \%$ of all ovarian neoplasms and $60 \%$ of all benign ovarian neoplasms [4-6]. Although these are common tumors, with gastrointestinal-type epithelium found in 7 $13 \%$ of the cases [7], the identification of organized

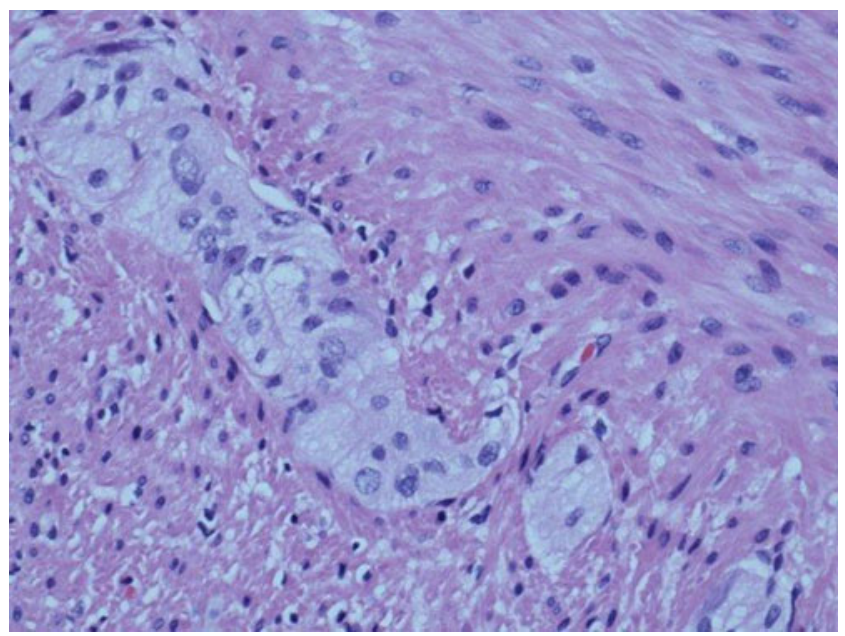

Fig. 4 Microscopically, the tubular structure with myenteric (Auerbach's) nerve plexus located in the muscularis propria 
gastrointestinal tissue, including muscular layers, is rare and identified usually only microscopically $[3,7,8]$. The case reported by Kwon et al. is the only reported macroscopic gastrointestinal tissue found within an ovarian tumor [2], and the case reported by Tang et al. is the only reported occurrence of a mature cystic teratoma of the ovary containing complete colonic wall in continuity with an endocervical-type mucinous cystadenoma [3]. Our case differs from the previous reports as the fully developed intestinal tissue was found to be extending from the mass.

At the time of surgery, we were initially uncertain whether this tissue represented normal bowel, fallopian tube, or other tissue, adherent to the mass or whether this tubular structure was actually part of the tumor. Bowel injury is a frequent concern in gynecologic surgery and complicates approximately $0.2-0.5 \%$ of cases $[9,10]$. This risk increases with adhesions from prior surgery, infection, or endometriosis [1]. Importantly, we were able to identify and completely excise the structure from the omentum while giving careful attention to not injure the "bowel." In addition, this case illustrates the need to recognize situations requiring conversion of laparoscopic surgery to laparotomy when facing a challenging case. We encourage careful evaluation and dissection in surgical cases involving extensive adhesive disease, and we recognize the risk of adhesive disease associated with a prior surgical history.

Conflict of interest We declare that we have no conflict of interest.

\section{References}

1. Schorge J, Schaffer J, Halvorson L, Hoffman B, Bradshaw K, Cunningham F (2008) Williams gynecology, 1st edn. McGrawHill, New York

2. Kwon MJ, Nam ES, Cho SJ, Shin HS, Na YS, Chang JH, Kim IS (2009) Bowel loop in an ovarian tumor: grossly visible, completely developed intestinal loop in mature cystic teratoma of malignant mixed germ cell tumor. Pathol Int 59(7):479-481

3. Tang P, Soukkary S, Kahn E (2003) Case report: mature cystic teratoma of the ovary associated with complete colonic wall and mucinous cystadenoma. Ann Clin Lab Sci 33(4):465-470

4. Katsube Y, Berg JW, Silverberg SG (1982) Epidemiologic pathology of ovarian tumors: a histopathologic review of primary ovarian neoplasms diagnosed in the Denver Standard Metropolitan Statistical Area, 1 July-31 December 1969 and 1 July-31 December 1979. Int J Gynecol Pathol 1(1):3-16

5. Koonings PP, Campbell K, Mishell DR Jr, Grimes DA (1989) Relative frequency of primary ovarian neoplasms: a 10-year review. Obstet Gynecol 74(6):921-926

6. Peterson WF, Prevost EC, Edmunds FT, Hundley JM Jr, Morris FK (1955) Benign cystic teratomas of the ovary: a clinicostatistical study of 1,007 cases with a review of the literature. Am J Obstet Gynecol 70(2):368-382

7. Fujiwara K, Ginzan S, Silverberg SG (1995) Mature cystic teratomas of the ovary with intestinal wall structures harboring intestinal- type epithelial neoplasms. Gynecol Oncol 56(1):97-101

8. Woodfield B, Katz DA, Cantrell CJ, Bogard PJ (1985) A benign cystic teratoma with gastrointestinal tract development. Am J Clin Pathol 83:236-240

9. Harris WJ (1997) Complications of hysterectomy. Clin Obstet Gynecol 40(4):928-938

10. Mäkinen J, Johansson J, Tomás C, Tomás E, Heinonen PK, Laatikainen T, Kauko M, Heikkinen AM, Sjöberg J (2001) Morbidity of 10110 hysterectomies by type of approach. Hum Reprod 16(7):1473-1478 